NASZA DERMATOLOGIA Online

OUR DERMATOLOGY Online

Source of Support:

Georgia Dermatopathology Associates, Atlanta, Georgia, USA

Competing Interests: None declared

\section{AN ALLERGIC BULLOUS DRUG REACTION TRIGGERED BY LEVOFLOXACIN AND TRIMETHOPRIM / SULFAMETHOXAZOLE MIMICKING AN AUTOIMMUNE BLISTERING DISEASE}

\author{
Ana Maria Abreu Velez ${ }^{1}$, A. Deo Klein ${ }^{2}$, Michael S. Howard ${ }^{1}$ \\ ${ }^{1}$ Georgia Dermatopathology Associates, Atlanta, Georgia, USA \\ ${ }^{2}$ Statesboro Dermatology, Statesboro, Georgia, USA
}

Corresponding author: Ana Maria Abreu Velez, MD PhD

abreuvelez@yahoo.com

\begin{abstract}
Introduction: Allergic drug reactions represent common clinical challenges. Selected allergic drug reactions present with blisters.

Case report: A 73 year old female was prescribed levofloxacin injection and trimethoprim/sulfamethoxazole for a urinary tract infection. Subsequently, the patient developed blisters on both of her hands and feet, associated with clinical pruritus. Clinically, bullae were observed on both of her palms and soles, associated with erythema.

Methods: Biopsies for hematoxylin and eosin (H\&E) examination, as well as for direct immunofluorescence (DIF) were performed.

Results: Focal areas of the epidermis displayed diffuse, mild spongiosis. An acute inflammatory process extended into hair follicular units, with focal rupture of these structures. Focal subepidermal vesiculations were also noted in the areas of follicular unit rupture. DIF examination demonstrated faint linear deposits of anti-human fibrinogen at the basement membrane zone of the skin, as well as around several hair shafts. A similar fibrinogen deposition pattern also present around the upper dermal blood vessels.

Conclusions: In our practice experience, the most common cause of blistering diseases are allergic drug reactions, in contradistinction to primary autoimmune blistering disorders. Clinical physicians, pathologists and immunodermatologists should be aware that allergic drug reactions can mimic primary autoimmune blistering disorders, both clinically and in selected immunologic aspects.
\end{abstract}

Key words: cutaneous allergic drug reaction; direct immunofluorescence; biomarkers; hair follicle; trimethoprim/sulfamethoxazole; levofloxacin

Abbreviations and acronyms: Immunohistochemistry (IHC), hematoxylin and eosin (H\&E), direct immunofluoresence (DIF), basement membrane zone (BMZ).

\section{Introduction}

Drug-induced allergic reactions are characterized by a spectrum of clinical and histologic patterns that include perivascular dermatitis, vesiculobullous lesions, pustular eruptions, sclerodermoid reactions, vasculitis, folliculitis/ perifolliculitis and panniculitis [1-12]. While a single drug may elicit a range of reaction patterns, no reaction pattern is specific for a particular drug. Although the temporal link between initiation of drug therapy and the onset of the drug rash is critical to the diagnosis, drug reactions may also occur during the course of chronic drug administration [1,12].

\section{Case report}

A 73 year old female was prescribed levofloxacin injection and trimethoprim/sulfamethoxazole for a tract urinary infection. Subsequently, the patient consulted a dermatologist for development of blisters on both of her hands and feet with pruritus. Clinically, small blisters on erythematous base were seen on both of her palms and soles. Skin biopsies for hematoxylin and eosin (H\&E) examination, and for direct immunofluorescence were taken in 10\% formalin and in Michel's media, respectively. Following the biopsies, the patient's cutaneous lesions improved over a period of weeks following discontinuation of her trimethoprim/sulfamethoxazole and initiation of oral prednisone. 
Methods

Direct immunofluorescence (DIF)

In brief, skin cryosections were prepared, and incubated with multiple fluorochromes as previously reported [3-8]. We utilized normal skin as a negative control, obtained from patients going under esthetic plastic surgery. To test the local immune response in lesional skin, we used antibodies to immunoglobulins A, G, M, and E, as well as Complement $\mathrm{C} 3$ and fibrinogen in DIF testing (all FITC conjugated, and all from Dako, Carpinteria, California). We also utilized monoclonal anti-collagen IV from Sigma (Saint Louis, Missouri, USA). We further utilized Texas red as a secondary DIF chromogen to further characterize the immune response within hair follicles.

\section{Results}

Examination of the H\&E tissue sections demonstrated an acute inflammatory process. Focal areas of the epidermis displayed diffuse, mild spongiosis. The dermis displayed congested blood vessels. An acute inflammatory process extended into hair follicular units, with focal rupture of these structures. Focal subepidermal vesiculations were also noted in the areas of follicular unit rupture. A superficial, moderately florid, mixed perivascular inflammatory infiltrate was present within the dermis, featuring lymphocytes, histiocytes, neutrophils and eosinophils. No vasculitis was appreciated. Focally, necrotic epidermal keratinocytes were observed (Fig. 1). Direct immunofluorescence (DIF) studies were performed, and displayed the following results: $\mathrm{IgG}$ (-); IgA (-); IgM (-); IgD (-); IgE (-); complement/C1q (-); complement/C3 $(+)$, accentuated around the upper dermal blood vessels; and collagen IV $(++)$ around hair follicles, sweat glands and along the basement membrane zone $(B M Z)$. Fibrinogen $(++)$ was noted in a faint linear pattern at the BMZ and around dermal superficial blood vessels (Fig. $1)$.

\section{Discussion}

The diagnosis of an allergic drug reaction is based on a detailed clinical history, and a temporal correlation between initiation of medication therapy and onset of the rash [1-3]. Histopathology aids in the diagnosis, and immunofluorescence characterizes the nature of immune deposits. In a recent study, the most common manifestations of a cutaneous drug eruption were a maculopapular rash; other possible clinical manifestations included purpura, acneiform lesions, TEN/Stevens Johnson syndrome, erythema multiforme, exfoliative dermatitis and other blistering reactions [11]. Associated drugs included nonsteroidal antinflammatory drugs, antipsychotics, antibiotics, antileprotics/antitubercular drugs, steroids, antimitotics and cardiac and renal specialty drugs. Histopathological features were compatible with the clinical lesions in most of the cases. The most common immunoreactants in direct immunofluorescence were complement/C3 and fibrinogen, primarily noted around dermal blood vessels and at the BMZ [11].

Clues to the drug-induced nature of our cutaneous eruption include the presence of overlapping, incongruent histologic reaction patterns, the clinical features, and immunologic features observed in the DIF. While eosinophils represent an important histologic hallmark of an allergic drug reaction, they may also be conspicuous in skin rashes without a drug association. Furthermore, eosinophils may be histologically sparse or absent in some allergic drug reactions. Thus, increased awareness of the broad spectrum of cutaneous pathology and direct immunofluorescence patterns elicited by an increasing range of therapeutic agents is critical to the proper diagnosis of these disorders. Significantly, in our case, the DIF results do not represent a classic pattern observed in any primary autoimmune blistering disease.

\section{REFERENCES}

1. Wintroub BU, Stern R: Cutaneous drug reactions: pathogenesis and clinical classification. J Am Acad Dermatol. 1985;13:167-79.

2. Stern RS, Wintroub BU: Adverse drug reactions: reporting and evaluating cutaneous reactions. Adv Dermatol. 1987;2:3-17.

3. Abreu-Velez AM, Klein AD, Smoller BR, Howard MS: Bullous allergic drug eruption with presence of myeloperoxidase and reorganization of the dermal vessels observed by using CD34 and collagen IV antibodies N Am J Med Sci. 2011;3:82-4.

4. Abreu-Velez AM, Howard MS, Smoller BR: Atopic dermatitis with possible polysensitization and monkey esophagus reactivity. N Am J Med Sci. 2010;2:336-40.

5. Abreu-Velez AM, Pinto FJ Jr, Howard MS: Dyshidrotic eczema: relevance to the immune response in situ. N Am J Med Sci. 2009; $1: 117-20$.

6. Abreu-Velez AM, Jackson BL, Howard MS: Deposition of immunoreactants in a cutaneous allergic drug reaction. N Am J Med Sci. 2009;1:180-3

7. Abreu-VelezAM, Smith JG Jr, Howard MS: Presence of neutrophil extracellular traps and antineutrophil cytoplasmic antibodies associated with vasculitides. N Am J Med Sci. 2009;1:309-13.

8. Abreu-Velez AM, Loebl AM, Howard MS: Spongiotic dermatitis with a mixed inflammatory infiltrate of lymphocytes, antigen presenting cells, immunoglobulins and complement. N Dermatol Online. 2011;2:52-7.

9. Abreu-Velez AM, Jackson BL, Howard MS: Salt and pepper staining patterns for LAT, ZAP-70 and MUM-1 in a vasculitic bullous allergic drug eruption. N Dermatol Online. 2011;2:104-7. 10. Abreu-Velez AM, Klein AD, Howard MS: LAT, EGFR -pY197, PCNL2, CDX2, HLA-DPDQDR, bromodeoxyuridine, JAM-A, and ezrin immunoreactants in a rubbed spongiotic dermatitis. Our Dermatol Online. 2011;2:211-5.

11. Sehgal S, Balachandran C, Shenoi SD: Clinical study of cutaneous drug reactions in 80 patients. Indian J Dermatol Venereol Leprol. 2003;69:6-7.

12. Ramdial PK, Naidoo DK: Drug-induced cutaneous pathology. J Clin Pathol. 2009;62:493-04. 


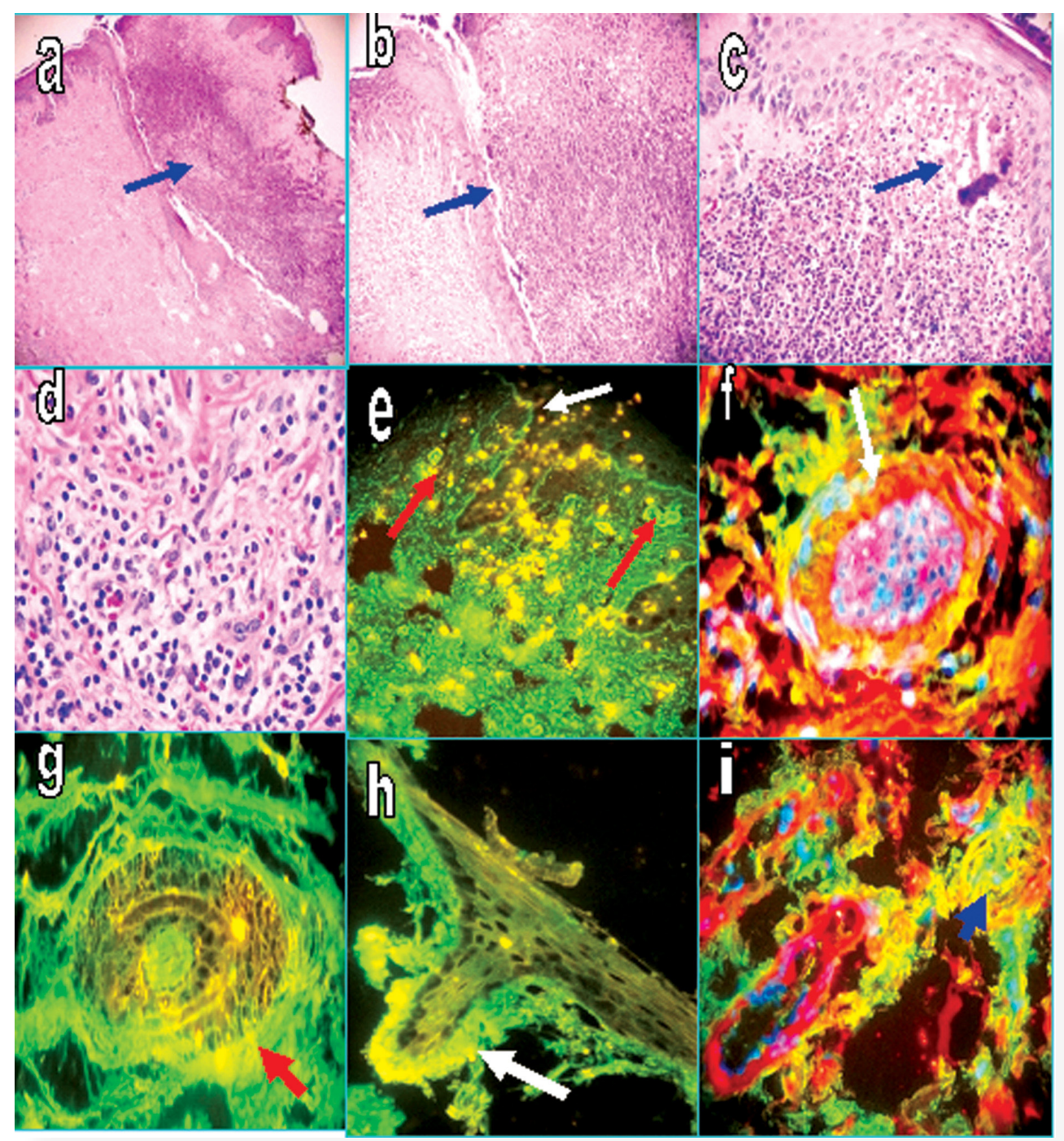

Figure 1. a through d, $\mathbf{H} \&$ E staining. In $\mathbf{a}$ and $\mathbf{b}$, note a blister cleft at the periphery of a hair follicle, and an early subepidermal blister cleft (blue arrows) (100x). The subjacent dermis features significant edema, as well as a superficial, perivascular mixed inflammatory infiltrate featuring plasma cells and eosinophils. c. Note the focal subepidermal blister, and a strong subjacent inflammatory infiltrate featuring lymphocytes, histiocytes, eosinophils and neutrophilic debris (200x). In $\mathbf{d}$, we highlight dermal edema, focal lymphocytic exocytosis and dermal extravasation of red blood cells within the infiltrate of 1c (400x). e Through $\mathbf{i}$, DIF. e. DIF showing faint basement membrane zone linear staining (green staining; white arrow) and also positive staining around the superficial dermal blood vessels (red arrows) using FITC conjugated anti-human fibrinogen. f. Shows a multicolor DIF. The white arrow highlights faint BMZ staining around a hair follicle shaft with FITC conjugated anti human fibrinogen (green-yellow staining). Background dermal collagen is stained with Texas red conjugated anti-collagen IV antibody (red staining). g. Similar to $\mathbf{f}$, but utilizing single color DIF and only the fibrinogen antibody (red arrow). $\mathbf{h}$. DIF showing a pathologic separation of the epidermis of the specimen, and focal subepidermal FITC conjugated fibrinogen reactivity (yellow staining; white arrow). i. Multicolor DIF showing two hair follicles, each separate from the adjacent dermis. Close to the follicles, please note that an adjacent nerve is positive for FITC conjugated anti-human fibrinogen (yellow staining, blue arrow). Background dermal collagen is stained with Texas red conjugated anti-collagen IV (red staining).

Copyright by Ana Maria Abreu Velez et al. This is an open access article distributed under the terms of the Creative Commons Attribution License, which permits unrestricted use, distribution, and reproduction in any medium, provided the original author and source are credited. 